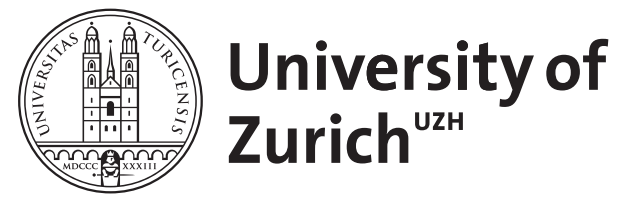

\title{
Harmonic sequence paradox
}

Blavatskyy, Pavlo R

\begin{abstract}
Informal evidence suggests that individuals are willing to pay only a finite and, typically, very low price for a specific lottery that converges to an infinite payment with probability one. The established decision theories (expected value, expected utility theory, cumulative prospect theory) cannot satisfactorily explain this low willingness to pay. The presented paradox strengthens the original and the super St. Petersburg paradox.
\end{abstract}

DOI: https://doi.org/10.1007/s00199-005-0606-9

Posted at the Zurich Open Repository and Archive, University of Zurich ZORA URL: https://doi.org/10.5167/uzh-2235

Journal Article

Published Version

Originally published at:

Blavatskyy, Pavlo R (2006). Harmonic sequence paradox. Economic Theory, 28(1):221-226.

DOI: https://doi.org/10.1007/s00199-005-0606-9 


\title{
Harmonic sequence paradox ${ }^{\star}$
}

\author{
Pavlo R. Blavatskyy \\ Institute for Empirical Research in Economics, University of Zurich, \\ Winterthurerstrasse 30, 8006 Zurich, SWITZERLAND \\ (e-mail: pavlo.blavatskyy@iew.unizh.ch)
}

Received: Spetember 27, 2004; revised version: January 15, 2005

Summary. Informal evidence suggests that individuals are willing to pay only a finite and, typically, very low price for a specific lottery that converges to an infinite payment with probability one. The established decision theories (expected value, expected utility theory, cumulative prospect theory) cannot satisfactorily explain this low willingness to pay. The presented paradox strengthens the original and the super St. Petersburg paradox.

Keywords and Phrases: Expected value, EUT, Cumulative prospect theory, St. Petersburg paradox, Willingness to pay.

\section{JEL Classification Numbers: C91, D81.}

Consider an urn that initially contains one white and one black ball. An individual draws one ball from this urn and receives one dollar (nothing) should the ball be white (black). Whatever the drawn ball happens to be, it is subsequently put back into the urn. Additionally, one more black ball is added to the urn. The individual then draws one ball again and the cycle continues ad infinitum. At each iteration, the drawn white ball pays off one dollar and the number of black balls is increased by one. What is a maximum price that a rational individual is willing to pay for participation in this lottery $L$ ?

Informal evidence suggests that an individual's willingness to pay (WTP) for lottery $L$ is finite and, typically, very low (less than ten dollars). This finding is paradoxical because it cannot be easily accommodated within established decision

* I am grateful to Peter Wakker, whose suggestions helped to simplify significantly the exposition of the main idea, and to the participants of a brown-bag seminar at CERGE-EI (June 23, 2004, Prague), notably Dirk Engelmann and Andreas Ortmann, who suggested interesting testable explanations for the paradox. 


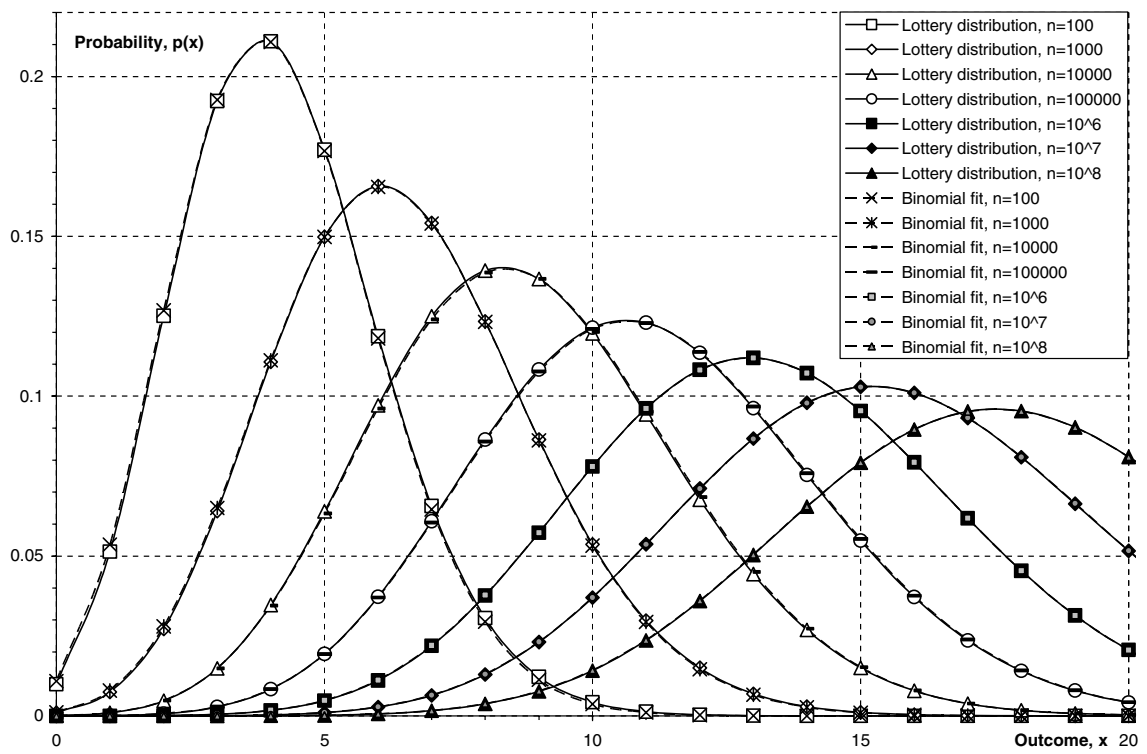

Figure 1. The true lottery distribution and its approximation by the binominal distribution. The points of a discrete distribution are connected by a smoothed continuous line to facilitate the distinction between different distributions

theories, as demonstrated below. Let $p_{n}(x)$ denote the probability of winning $x \in$ $[0, n]$ dollars after $n$ draws of a ball. Equation (1) defines the lottery distribution $p_{n}:[0, n] \rightarrow[0,1]$ recursively. For large $n$, the lottery distribution $p_{n}$ has mean $\mu_{n}=1 / 2+1 / 3+\ldots+1 / n \cong \ln n-1+C_{1},{ }^{1}$ where $C_{1}=0.5772 \ldots$ is the EulerMascheroni constant, and variance $\sigma_{n}^{2} \cong \ln n-C_{2}$, where $C_{2}=1.0676 \ldots$ is a constant. For large $n$, the lottery distribution $p_{n}$ can be accurately approximated by the binomial distribution $B_{m}(q)$ that has the same mean $\left(m q=\mu_{n}\right)$ and variance $\left(m q(1-q)=\sigma_{n}^{2}\right)$, as demonstrated by Figure 1 .

$$
\begin{aligned}
& p_{n}(0)=1 /(n+1) \\
& p_{n}(n)=1 /(n+1) ! \\
& p_{n}(x)=p_{n-1}(x) \cdot n /(n+1)+p_{n-1}(x-1) \cdot 1 /(n+1), x \in[1, n-1]
\end{aligned}
$$

Clearly, the concept of expected value (EV) is not a descriptive decision theory for lottery $L$ because it predicts an infinitely large WTP for $L\left(\lim _{n \rightarrow \infty} \mu_{n}=\infty\right)$. This conclusion restates the implication of the original St. Petersburg paradox (e.g. Bernoulli, 1738).

Proposition 1. For any finite monetary value $x$, the probability that lottery $L$ delivers $x$ or less is zero $\left(\forall x \in \mathbb{N} \cup\{0\}: \lim _{n \rightarrow \infty} \sum_{y=0}^{x} p_{n}(y)=0\right)$.

Proof of Proposition 1 is presented in Appendix.

1 Sequence $1 / 2,1 / 3, \ldots, 1 / n$ is called the harmonic sequence, which explains the name of the paradox 
Proposition 1 effectively states that an outcome of lottery $L$ converges to an infinite payment with certainty. In other words, the reduced form of the compound representation of $L$ (in the first paragraph) is an infinite payment with probability one.

Expected utility theory, or EUT, (e.g. von Neumann and Morgenstern, 1944) with unbounded utility function (e.g. $u(x)=\ln x, u(x)=\sqrt{x}$ ) cannot explain a finite WTP for $L$. The concept of unbounded EUT is not a descriptive decision theory for lottery $L$ because it predicts an infinitely large WTP for $L\left(u^{-1}(E U T(L))=\right.$ $\left.u^{-1}(u(\infty) \cdot 1)=\infty\right)$. This conclusion restates the implication of the so-called super St. Petersburg paradox (e.g. Menger, 1934).

EUT with a bounded utility function can explain a finite WTP for $L$ only if there exists a finite wealth level $M$ beyond which the marginal utility of any additional income is zero $(\exists M \in \mathbb{N}: \forall x \geq M, u(x)=u(M))$. This is a stronger restriction than the one imposed by the super St. Petersburg paradox, which requires a utility function just to be bounded $(\exists W \in \mathbb{R}: \forall x \in \mathbb{N}, u(x) \leq W$ ). An individual's WTP for $L$ then is $u^{-1}(\operatorname{EUT}(L))=u^{-1}(u(\infty) \cdot 1)=u^{-1}(u(M))=M$. When an observed WTP for $L$ is of a magnitude of ten dollars, according to the bounded EUT, an individual's marginal utility of any income above ten dollars should be zero. Such implication clearly contradicts common sense and the existing experimental evidence (e.g. Wakker and Deneffe, 1996). Thus, the EUT with a bounded utility function can explain a finite WTP for $L$ but it cannot satisfactorily explain a low WTP for $L$. The same conclusion holds for rank-dependent utility theories (allowing for a continuous non-linear probability weighting function) and the most prominent of them - the cumulative prospect theory, or CPT, (e.g. Tversky and Kahneman, 1992).

Apparently, none of the established decision theories (EV, EUT, CPT) can satisfactorily explain the harmonic sequence paradox. Figure 2 illustrates this theoretical conclusion. For different (large) numbers of iterations, an individual's WTP for $L$ is calculated as predicted by EV, EUT and CPT. The true lottery distribution $p_{n}$ is approximated by the binomial distribution. All theories predict that WTP is increasing by approximately 2 dollars with a tenfold increase in the number of iterations. EV (a solid line) predicts the highest WTP. The prediction of EUT (dashed lines) with unbounded utility functions $u(x)=\sqrt{x}, u(x)=x^{0.88}$ is approximately 50 cents below the prediction of EV. The prediction of CPT (dotted lines) is almost identical for four different parameterizations that were elicited by Tversky and Kahneman (1992), Wu and Gonzalez (1996), Abdellaoui (2000), and Bleichrodt and Pinto (2000). CPT predicts the lowest WTP for $L$, which is approximately 2 dollars below the prediction of EV.

\section{Conclusion}

The presented paradox employs a specific lottery $L$ converging to an infinite payment with certainty. This fact notwithstanding, informal evidence suggests that individuals are willing to pay only a finite and low price for $L$. The established decision theories (EV, EUT, CPT) cannot satisfactorily explain this low WTP. The presented paradox is stronger evidence against expected value (unbounded EUT) 


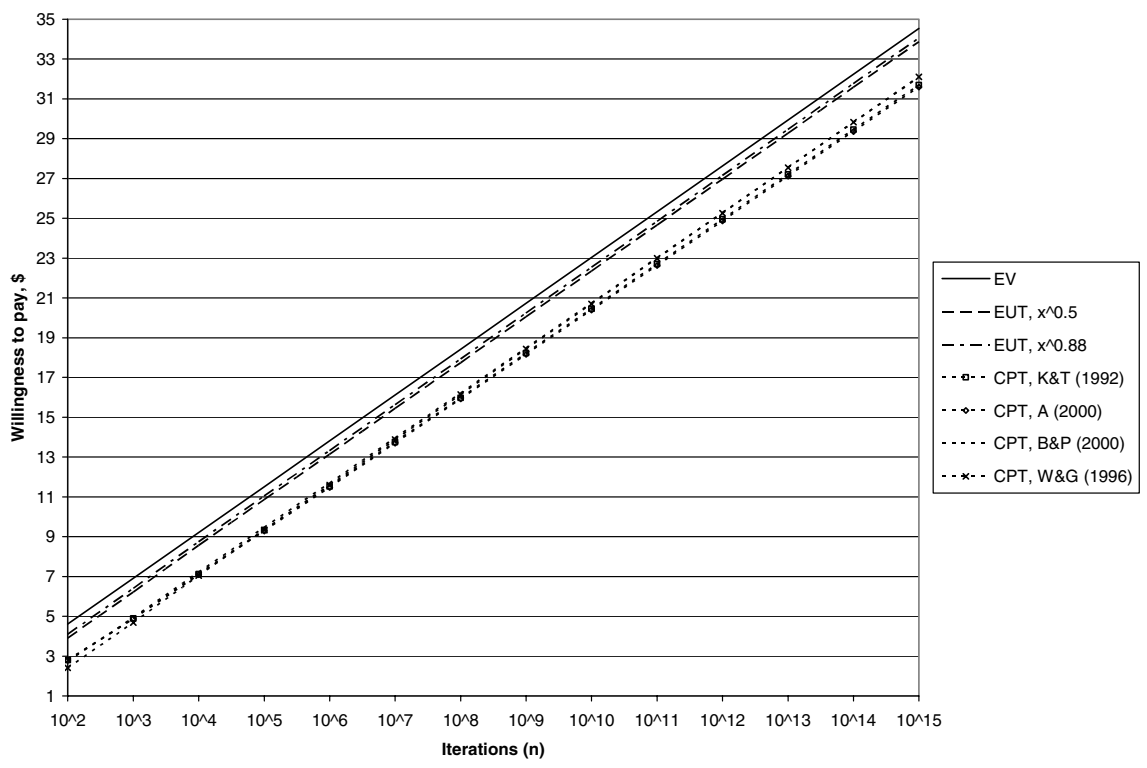

Figure 2. An individual willingness to pay (in dollars) for the lottery employed in the harmonic sequence paradox (as a function of a number of iterations) calculated through: a) the expected value (EV); b) the expected utility theory (EUT), with individual utiltity function $u(x)=\sqrt{x}, u(x)=x^{0,88}$; c) the cumulative prospect theory (CPT), with parameters as in Tversky and Kahneman (1992), Wu and Gonzalez (1996), Abdellaoui (2000), and Bleichrodt and Pinto (2000)

than the original (super) St. Petersburg paradox. The St. Petersburg paradoxes employ a lottery with exponentially growing outcomes that can quickly exceed the finite wealth of the planet (e.g. Samuelson, 1977, p. 27). The presented paradox employs lottery $L$ whose explicit outcome is just one dollar per iteration. Thus, an individual is psychologically more averse to $L$ compared to a lottery with explicitly mentioned large outcomes (as in the St. Petersburg paradoxes). The implicit outcomes of lottery $L$ grow logarithmically with the number of iterations.

The proposed paradox may be refuted on practical grounds. Since it employs a lottery with logarithmically growing mean, about $10^{43}$ iterations are required to guarantee at least a modest expected outcome of 100 dollars. This problem is not so apparent in the St. Petersburg paradoxes where the probability of infinitely many iterations is infinitely small. However, an individual may be informed that $L$ is simulated on a computer with high processing speed for a finite (large) number of iterations (e.g. $10^{15}$ ) within a short period of time (e.g. 5 minutes). Any observed discrepancy between actual WTP (e.g. $<\$ 10)$ and the theoretical prediction (e.g. $>\$ 30$ as demonstrated by Fig. 2) is then as damaging to the established decision theories as the infinite version of the paradox itself. Such discrepancy also rules out the explanation of a low WTP because an individual either anticipates only a finite (low) number of iterations or may become bored after many iterations. By eliciting an individual's evaluation of the sum of the harmonic sequence $\sum_{i=2}^{\infty} 1 / i$ it is possible to test yet another explanation of a low WTP for $L$ due to an individual's failure to realize that this sum is diverging. Finally, this theoretical note can be 
complemented with the experimental elicitation of an individual's WTP for a finite $L$ when there are several white balls in the urn initially (or the number of white balls, or the monetary payoff on a white ball, increases slightly with the number of iterations). Such experimental results may identify the conditions under which the discrepancy between actual and predicted WTP decreases.

\section{Appendix}

\section{Proof of Proposition 1}

Lemma 1. For any finite monetary value $x$, the probability that lottery $L$ delivers outcome $x$ is zero $\left(\forall x \in \mathbb{N} \cup\{0\}: \lim _{n \rightarrow \infty} p_{n}(x)=0\right)$.

Proof by contradiction. Suppose that $\exists x \in \mathbb{N} \cup\{0\}: \lim _{n \rightarrow \infty} p_{n}(x) \neq 0$. Using formula (1), $p_{n}(x+1)=\frac{n}{n+1} p_{n-1}(x+1)+\frac{1}{n+1} p_{n-1}(x)=$ $\frac{n}{n+1}\left(\frac{n-1}{n} p_{n-2}(x+1)+\frac{1}{n} p_{n-2}(x)\right)+\frac{1}{n+1} p_{n-1}(x)=\ldots=$ $\frac{1}{n+1}\left(p_{x}(x)+\ldots+p_{n-2}(x)+p_{n-1}(x)\right)$. Since $\lim _{n \rightarrow \infty} p_{n}(x) \neq 0 \Rightarrow \exists \varepsilon>$ $0: \forall n \geq x, p_{n}(x) \geq \varepsilon$. Then, $p_{n}(x+1) \geq \frac{n-x}{n+1} \varepsilon \Rightarrow \lim _{n \rightarrow \infty} p_{n}(x+1) \geq \varepsilon$. Similarly, it is possible to prove that $\forall k \in \lim _{n \rightarrow \infty} p_{n}(x+k) \geq \varepsilon$. Then, $\lim _{n \rightarrow \infty} \sum_{y=0}^{n} p_{n}(y) \geq \lim _{n \rightarrow \infty} \sum_{y=x}^{n} p_{n}(y) \geq \lim _{n \rightarrow \infty}(n-x+1) \varepsilon=\infty$. However, this contradicts the assumption that $p_{n}$ is a probability distribution i.e. $\lim _{n \rightarrow \infty} \sum_{y=0}^{n} p_{n}(y)=1$

Proposition 1 is proved by mathematical induction.

a) When $x=0$, Proposition 1 holds trivially: $\lim _{n \rightarrow \infty} \sum_{y=0}^{x} p_{n}(y)=$ $\lim _{n \rightarrow \infty} p_{n}(0)=\lim _{n \rightarrow \infty} \frac{1}{n+1}=0$.

b) Suppose that Proposition 1 holds for $\forall x<X$.

c) Let us prove that Proposition 1 then also holds for $x=X$. $\lim _{n \rightarrow \infty} \sum_{y=0}^{X} p_{n}(y)=\underbrace{\lim _{n \rightarrow \infty} p_{n}(X)}_{=0 \text { due to Lemma } 1}+\underbrace{\lim _{n \rightarrow \infty} \sum_{y=0}^{X-1} p_{n}(y)}_{=0 \text { due to assumption b) }}=0$.

Proposition 1 then holds for any finite $x$ by mathematical induction.

\section{References}

Abdellaoui, M.: Parameter-free elicitation of utility and probability weighting functions. Management Science 46, 1497-1512 (2000)

Bernoulli, D.: Specimen theoriae novae de mensura sortis, Commentarii Academiae Scientiarum Imperialis Petropolitanae. (1738) Translated by Bernoulli, D.: Exposition of a new theory on the measurement of risk. Econometrica 22, 23-36 (1954)

Bleichrodt, H., Pinto, J.: A parameter-free elicitation of the probability weighting function in medical decision analysis. Management Science 46, 1485-1496 (2000)

Menger, K.: Das Unsicherheitsmoment in der Wertlehre. Betrachtungen im Anschluss an das sogenannte Petersburger Spiel. Zeitschrift fur Nationaloconomie 5, 459-485 (1934)

Samuelson, P.: St. Petersburg paradoxes: defanged, dissected, and historically described. Journal of Economic Literature 15, 24-55 (1977)

Tversky, A., Kahneman, D.: Advances in prospect theory: cumulative representation of uncertainty. Journal of Risk and Uncertainty 5, 297-323 (1992) 
von Neumann, J., Morgenstern, O.: Theory of games and economic behavior. Princeton, NJ: Princeton University Press 1944

Wakker, P.P., Deneffe, D.: Eliciting von Neumann-Morgenstern utilities when probabilities are distorted or unknown. Management Science 42, 1131-1150 (1996)

Wu, G., Gonzalez, R.: Curvature of the probability weighting function. Management Science 42, 16761690 (1996) 